OPEN ACCESS

Edited and reviewed by: Ramon Rios,

University of Southampton,

United Kingdom

${ }^{*}$ Correspondence:

Christian Mang

c.mang@ac-discovery.com Andrea Basso

andrea.basso@unige.it

Specialty section:

This article was submitted to

Organic Chemistry

a section of the journal

Frontiers in Chemistry

Received: 26 September 2018 Accepted: 03 October 2018

Published: 19 October 2018

Citation:

Moni L, De Moliner F, Garbarino S, Saupe J, Mang C and Basso A (2018) Corrigendum: Exploitation of the Ugi 5-Center-4-Component Reaction (U-5C-4CR) for the Generation of

Diverse Libraries of Polycyclic

(Spiro)Compounds.

Front. Chem. 6:510.

doi: 10.3389/fchem.2018.00510

\section{Corrigendum: Exploitation of the Ugi 5-Center-4-Component Reaction (U-5C-4CR) for the Generation of Diverse Libraries of Polycyclic (Spiro)Compounds}

\author{
Lisa Moni ${ }^{1}$, Fabio De Moliner ${ }^{1}$, Silvia Garbarino ${ }^{1}$, Jörn Saupe ${ }^{2}$, Christian Mang ${ }^{2 *}$ and \\ Andrea Basso ${ }^{1 *}$ \\ ${ }^{1}$ Dipartimento di Chimica e Chimica Industriale, Università degli Studi di Genova, Genova, Italy, ${ }^{2}$ AnalytiCon Discovery \\ GmbH, Potsdam, Germany
}

Keywords: multicomponent reactions, diversity oriented synthesis, scaffold diversity, combinatorial libraries, isocyanides

\section{A Corrigendum on}

Exploitation of the Ugi 5-Center-4-Component Reaction (U-5C-4CR) for the Generation of Diverse Libraries of Polycyclic (Spiro)Compounds

by Moni, L., De Moliner, F., Garbarino, S., Saupe, J., Mang, C., and Basso, A. (2018). Front. Chem. 6:369. doi: 10.3389/fchem.2018.00369

In the original article, there was an error. The sentence "The contemporary presence of a secondary amino group and a cyclic ketone kinetically disfavored the Ugi reaction, which did not proceed at room temperature, even with the addition of a Lewis acid as previously reported by Dawidowski" was misleading.

A correction has been made to Results and Discussion, Second paragraph

The contemporary presence of a secondary amino group and a cyclic ketone kinetically disfavored the Ugi reaction, which did not proceed at room temperature, even with the addition of a Lewis acid as previously reported by Dawidowski for other substrates (Dawidowski et al., 2014). However, by performing it in a sealed tube at $65^{\circ} \mathrm{C}$, a mixture of Ugi adduct 1 and cyclic imide 2 was isolated after 6 days. This mixture was subjected to complete cyclization by addition of a catalytic amount of DBU in acetonitrile. The overall yield after the two steps was an acceptable $47 \%$ and remarkably, under these conditions, no epimerization was observed at the L-proline $\alpha$-carbon, thus allowing us to obtain compound $\mathbf{2}$ in enantiomerically and diastereomerically pure form.

The authors apologize for this error and state that this does not change the scientific conclusions of the article in any way.

The original article has been updated. 


\section{REFERENCES}

Dawidowski, M., Sobczak, S., Wilczek, M., Kulesza, A., and Turło, J. (2014). Expanding the substrate scope of ugi five-center, four-component reaction (U5C-4CR): ketones as coupling partners for secondary amino acids. Mol. Divers. 18, 61-77. doi: 10.1007/s11030-013-9488-0

Conflict of Interest Statement: The authors declare that the research was conducted in the absence of any commercial or financial relationships that could be construed as a potential conflict of interest.
Copyright () 2018 Moni, De Moliner, Garbarino, Saupe, Mang and Basso. This is an open-access article distributed under the terms of the Creative Commons Attribution License (CC BY). The use, distribution or reproduction in other forums is permitted, provided the original author(s) and the copyright owner(s) are credited and that the original publication in this journal is cited, in accordance with accepted academic practice. No use, distribution or reproduction is permitted which does not comply with these terms. 